\title{
Comparação de modelos matemáticos na estimativa das perdas de produtividade de grãos em arroz irrigado
}

\author{
Dirceu Agostinetto ${ }^{(1)}$, Nilson Gilberto Fleck ${ }^{(1)}$, Mauro Antonio Rizzardi ${ }^{(1)}$, Mário Antônio Bianchi( ${ }^{(1)}$ \\ e Paulo Régis Ferreira da Silva(1) \\ (1)Universidade Federal do Rio Grande do Sul, Fac. de Agronomia, Caixa Postal 15100, CEP 90001-970 Porto Alegre, RS. \\ E-mail: agostinetto@agricultura.gov.br, fleck@ufrgs.br, rizzardi@zaz.com.br, mario.bianchi@ig.com.br, paulo.silva@ufrgs.br
}

\begin{abstract}
Resumo - Os objetivos deste trabalho foram comparar modelos matemáticos e identificar, entre variáveis morfológicas, a de melhor ajuste na previsão de perdas de produtividade em arroz irrigado por interferência da cultivar EEA 406, simuladora de arroz-vermelho. Foram realizados quatro experimentos, sendo um no campo e os demais em casa de vegetação. Três cultivares de arroz, BRS-38 Ligeirinho, IRGA 417 e BR-IRGA 409, foram estudadas no campo, com espaçamento entrelinhas de 15 e $25 \mathrm{~cm}$, além de populações da cultivar competidora (dez níveis). Em casa de vegetação, realizaram-se experimentos em monocultivos e em série de substituição. A análise dos dados foi realizada com aplicação de modelos lineares e não lineares de regressão. O melhor ajuste dos dados de perdas de produtividade em arroz foi encontrado com o modelo de dois parâmetros. Área foliar e cobertura do solo estimaram melhor as perdas de produtividade de grãos do que a massa seca da cultivar simuladora. Os modelos testados indicam que a redução do espaçamento entrelinhas aumenta a habilidade competitiva das cultivares de arroz em relação à cultivar concorrente.
\end{abstract}

Termos para indexação: Oryza sativa, cultivares de arroz, espaçamento entrelinhas.

\section{Comparison of mathematical models in estimating flooded rice grain yield losses}

\begin{abstract}
The objectives of this work were to compare mathematical models and identify, among morphologic variables, the one better adjusted to rice grain yield losses by interference of EEA 406 cultivar, simulating red rice. Four experiments were carried out, one in the field and the others in a greenhouse. Rice cultivars BRS-38 Ligeirinho, IRGA 417, and BR-IRGA 409, were studied in the field in row widths of 15 and $25 \mathrm{~cm}$, and populations (ten levels) of concurrent cultivar. In greenhouse, tests in monocultures were carried out in a replacement series model. Data analysis were accomplished with application of linear and non linear regression models. The best adjustment of rice grain yield losses data was attained using the model with two parameters. Leaf area and soil coverage estimated better grain yield loss than dry weight of the simulating cultivar. The models show that reduction of crop row width increases the competitive ability of rice cultivars in relation to the concurrent genotypes.
\end{abstract}

Index terms: Oryza sativa, rice cultivars, row width.

\section{Introdução}

A estimativa do resultado da competição entre cultura e plantas daninhas por modelos de regressão, que integram tanto a população como a época de emergência das plantas daninhas, não considera o vigor relativo da cultura em relação às plantas daninhas, fator decisivo na compreensão da relação competitiva entre as espécies (Lutman et al., 1996). Além disso, por serem puramente matemáticos, os parâmetros desses modelos se referem às condições do experimento dos quais foram derivados e não são baseados nos processos bio- lógicos da competição. A fim de minimizar este efeito, Kropff \& Spitters (1991) propuseram um modelo que relaciona a perda de produtividade com parâmetros de crescimento como a área foliar relativa (AFR) das plantas daninhas pouco tempo após a emergência da cultura, usando o coeficiente de dano relativo (q) como único parâmetro do modelo. $\mathrm{O}$ parâmetro q representa o índice de competitividade das plantas daninhas sobre a cultura, isto é, quanto maior for seu valor, maior é a competitividade da planta daninha sobre a cultura e, deste modo, maior será a perda de produtividade da cultura (Chikoye \& Swanton, 1995; Knezevic et al., 1995). 
Posteriormente, Lotz et al. (1996) propuseram uma versão modificada do modelo de Kropff \& Spitters (1991), no qual incluíram a perda máxima de produtividade (m). A inclusão deste parâmetro, que limita a perda máxima de produtividade em $100 \%$, delimita uma clara assíntota para a curva hiperbólica (Chikoye \& Swanton, 1995). O modelo de dois parâmetros, o qual inclui a perda máxima de produtividade, forneceu melhores ajustes aos dados, quando comparado com o modelo de um único parâmetro (Chikoye \& Swanton, 1995; Knezevic et al., 1995).

Variáveis explicativas como área foliar, cobertura de solo e massa seca, podem ser usadas em substituição à população e à época de emergência das plantas daninhas, buscando estimar a interferência dessas variáveis em relação à cultura. Essas variáveis, quando relacionadas às correspondentes avaliadas na cultura, podem comparar o desenvolvimento das espécies e atuar como indicadoras do tamanho relativo, da produtividade e da capacidade fotossintética das espécies, que podem influenciar sua habilidade competitiva (Radosevich et al., 1997).

O poder competitivo de uma espécie está fortemente associado à sua participação na área foliar, no momento em que o dossel da população se fecha e inicia-se a competição interespecífica. Em razão disso, Kropff \& Spitters (1991), sugeriram usar a AFR na época apropriada para o controle de plantas daninhas em pós-emergência como um previsor da perda de produtividade da cultura, particularmente em situações em que a competição seja principalmente por luz. Modelos baseados na AFR das plantas daninhas têm apresentado elevada capacidade descritiva das perdas de produtividade de culturas em decorrência da interferência de plantas daninhas, em experimentos realizados no campo (Dieleman et al., 1995; Knezevic et al., 1995; Lotz et al., 1996).

Existe uma estreita relação entre perda de produtividade e cobertura foliar relativa (CFR) de plantas daninhas, medida pouco após a emergência da cultura, quando o dossel cultura-plantas daninhas ainda não está fechado (Kropff \& Spitters, 1991; Ngouajio et al., 1998). A CFR é, em relação a outras variáveis, fácil de determinar e representa uma estimativa adequada do crescimento da planta. Ela pode ser medida diretamente no campo de forma subjetiva (visualmente) ou objetiva (mecânica ou fotograficamente) (Lutman et al., 1996).

A produção de massa seca relativa (MSR) pelas plantas daninhas é outra variável explicativa que também pode ser usada para predizer as perdas de produtividade. Lutman et al. (2000) observaram que as previsões de perda de produtividade em canola, com base na massa relativa de plantas de esparguta (Stellaria media) variaram menos que aquelas que utilizaram a população da infestante. Outra vantagem é que as diferenças entre anos, nos valores de MSR, foram inferiores às encontradas nas populações de plantas daninhas.

A utilização de modelos matemáticos que consideram características morfológicas das plantas poderá melhorar a quantificação da interferência de plantas daninhas e, quando as variáveis forem relativas, aumentar a precisão das estimativas de perdas de produtividade da cultura, permitindo maiores índices de acerto nas decisões sobre controle de plantas daninhas.

Os objetivos deste trabalho foram comparar modelos matemáticos e identificar, entre variáveis morfológicas, a de melhor ajuste na previsão de perdas de produtividade por interferência causada pela cultivar EEA 406, simulando infestação de arroz-vermelho.

\section{Material e Métodos}

Foram realizados quatro experimentos, sendo um no campo e os demais em casa de vegetação.

A pesquisa no campo desenvolveu-se na Estação Experimental do Arroz, pertencente ao Instituto RioGrandense do Arroz (IRGA), Município de Cachoeirinha, RS, durante a estação de crescimento 2000/2001. O delineamento experimental utilizado foi o completamente casualizado, com uma unidade experimental por combinação de fatores. Os tratamentos, dispostos em esquema fatorial, constaram de três cultivares de arroz (BRS-38 Ligeirinho, ciclo muito curto; IRGA 417, ciclo curto; e, BR-IRGA 409, ciclo médio), de dois espaçamentos entrelinhas (15 e $25 \mathrm{~cm}$ ) e de 10 populações da cultivar EEA 406, a qual, por apresentar características morfológicas semelhantes às do arroz-vermelho, exerceu a função de simuladora do arroz daninho.

O experimento foi implantado em sistema convencional de preparo do solo. A área de cada parcela era de $9 \mathrm{~m}^{2}$. A semeadura foi realizada em 17/11/2000 e a emergência ocorreu sete dias após. As demais práticas de manejo foram realizadas de acordo com as recomendações para a cultura (Embrapa Clima Temperado, 1999).

As variáveis explicativas foram avaliadas aos $14 \mathrm{e}$ 28 dias após a emergência (DAE). A cobertura foliar 
do solo resultante da cultura e da cultivar simuladora foi avaliada de forma visual, por dois avaliadores, mediante atribuição de notas variáveis entre 0 e 100, para solo descoberto e totalmente coberto, respectivamente. A determinação da massa seca foi realizada por meio de colheita e secagem em estufa, até peso constante, da parte aérea das plantas presentes em área de $0,125 \mathrm{~m}^{2}$ por parcela. A área foliar foi quantificada com auxílio de integrador eletrônico de área foliar, utilizando-se as plantas que foram coletadas para determinação da massa seca.

Para determinar a produtividade de grãos, as panículas da área central de cada parcela $\left(4,2\right.$ e $3,5 \mathrm{~m}^{2}$ para espaçamento de 15 e $25 \mathrm{~cm}$, respectivamente) foram colhidas, quando o teor de umidade dos grãos de cada cultivar encontrava-se em torno de $22 \%$. Após a pesagem dos grãos e a correção do teor de umidade para $13 \%$, calcularam-se as perdas porcentuais de produtividade, em relação às parcelas mantidas livres de infestação.

$\mathrm{Na}$ avaliação da existência ou não de interações dos fatores estudados, realizou-se análise de variância dos dados de perdas porcentuais da produtividade de grãos, adotando-se como limites de aceitação o nível de 5\% de probabilidade para significância de efeitos individuais dos fatores e o de $15 \%$ para efeitos de interações simples. Assim, após ser verificada significância estatística, as relações entre dados de perdas de produtividade e as variáveis explicativas foram determinadas separadamente para cada cultivar e espaçamento entrelinhas, ajustando-se os dados aos modelos de regressão não linear da hipérbole retangular, propostos, respectivamente, por Cousens (1985) (Equação 1), Kropff \& Spitters (1991) (Equação 2) e Lotz et al. (1996) (Equação 3):

$\operatorname{Pr}=\mathrm{iX} /[1+(\mathrm{i} / \mathrm{a}) \mathrm{X}]$

$\operatorname{Pr}=100 \mathrm{qX} /[1+(\mathrm{q}-1) \mathrm{X}]$

$\operatorname{Pr}=100 \mathrm{qX} /[1+((\mathrm{q} / \mathrm{m})-1) \mathrm{X}]$

em que Pr é a perda (\%) de produtividade; X é a massa seca, área foliar ou cobertura foliar relativas; i é a perda de produtividade (\%) por unidade da competidora quando a respectiva variável se aproxima de zero; a é a perda de produtividade (\%) quando a variável em questão tende ao infinito; q é o coeficiente de dano relativo, estimado pelo modelo; e, m é a perda máxima da produtividade relativa, estimada pelo modelo.

$\mathrm{Na}$ aplicação da Equação 1, os valores de cobertura do solo (\%), massa seca da parte aérea $\left(\mathrm{g} \mathrm{m}^{-2}\right)$ e área foliar $\left(\mathrm{cm} \mathrm{m}^{-2}\right)$ foram multiplicados por 100, evitando-se assim a necessidade de correções desses valores no modelo. No cálculo das contribuições relativas (Equações 2 e 3) das variáveis cobertura foliar do solo, massa seca e área foliar, dividiu-se o valor da variável correspondente à simuladora pelo valor total da população.

$\mathrm{O}$ ajuste dos dados aos modelos foi realizado pelo procedimento Proc Nlin do programa computacional SAS (SAS Institute, 1989). Nos procedimentos de cálculos, utilizou-se o método de Gauss-Newton, o qual, por sucessivas iterações, estima os valores dos parâmetros nos quais a soma dos quadrados dos desvios das observações, em relação aos valores ajustados, seja mínima (Ratkowsky, 1983). Nos casos em que a estimativa do parâmetro a da Equação 2 foi superestimada, o valor da assíntota foi limitado para $100 \%$. Esse procedimento é recomendado para evitar perdas de produtividade superiores a $100 \%$, as quais são biologicamente irreais (Yenish et al., 1997; Askew \& Wilcut, 2001). O valor da estatística F, a 5\% de probabilidade, foi utilizado como critério de ajuste dos dados ao modelo. O critério de aceitação do melhor ajuste dos dados ao modelo baseou-se tanto no coeficiente de determinação $\left(\mathrm{R}^{2}\right)$ como na soma de quadrados do resíduo (SQR), de modo que maior valor do primeiro e menor valor do segundo representavam ajustes mais satisfatórios.

A comparação entre modelos que utilizaram variáveis relativas nas estimativas foi realizada por meio do teste F, conforme sugerido por Lotz et al. (1996) (Equação 4):

$\mathrm{F}=[\mathrm{SQR}(2)-\mathrm{SQR}(3)] /[\mathrm{SQR}(3) / \mathrm{GLr}(3)]$

em que F é o teste F; $\mathrm{SQR}(2$ e 3) são as somas de quadrados dos resíduos das Equações 2 e 3, respectivamente; GLr(3) são graus de liberdade do resíduo na Equação 3.

O modelo original de população (Cousens, 1985) não permite ordenação hierárquica, como ocorre com os modelos de variáveis relativas (Lotz et al., 1996), e por isso, as comparações entre as Equações 1 e 3 foram realizadas com base nas SQR. Assim, foi aceito como melhor modelo aquele que apresentasse menor SQR. Adicionalmente, para auxiliar a identificação do melhor modelo, realizou-se análise de correlação entre os valores de $\mathrm{R}^{2}$ e $\mathrm{SQR}$.

Os bioensaios foram instalados em 2001, em casa de vegetação, junto à sede da Faculdade de Agronomia da UFRGS, em Porto Alegre, RS. O primeiro bioensaio teve por objetivo determinar a produtividade final constante de arroz. Nesse caso, a produção da variável passa a ser independente da população de plantas. 
Os tratamentos constaram da cultivar de arroz IRGA 417 e de um biótipo de arroz-vermelho, cultivados em monocultivos nas populações equivalente a 158 , $315,472,630,787,945,1.102$ e 1.260 plantas $\mathrm{m}^{-2}$. As variáveis, avaliadas aos $31 \mathrm{DAE}$, foram massa seca das partes aérea e radical e área foliar.

O segundo bioensaio foi realizado com o objetivo de avaliar a área foliar dos concorrentes em condição de monocultivos. Os tratamentos constaram das cultivares IRGA 417 e EEA 406, cultivadas isoladamente nas populações de $26,53,105,211,421,842$ plantas $\mathrm{m}^{-2}$. As variáveis avaliadas, aos $30 \mathrm{DAE}$, foram massa seca da parte área e área foliar.

O terceiro bioensaio foi realizado no modelo série de substituição com o objetivo de estimar equações lineares recíprocas para determinar o parâmetro q. Os tratamentos constaram de diferentes combinações das cultivares IRGA 417 e EEA 406, variando-se as proporções de plantas por vaso $(0: 24 ; 6: 18 ; 12: 12 ; 18: 6$; 24:0), mantendo-se constante a população total de plantas $\left(945\right.$ plantas $\left.\mathrm{m}^{-2}\right)$. As avaliações, realizadas aos 32 DAE, constaram de massa seca da parte aérea e área foliar.

Aos dados de massa seca do bioensaio realizado em série de substituição, aplicou-se análise de regressão linear, utilizando-se o recíproco da produção de massa seca por planta como variável dependente e o recíproco da população de plantas como variável independente. Para se obter os parâmetros q e m, adotou-se a análise proposta por Kropff \& Spitters (1991) (Equações 5 e 6):

$\mathrm{q}=\left(\mathrm{b}_{\mathrm{cpd}} / \mathrm{b}_{\mathrm{c}}\right)\left(\mathrm{AF}_{\mathrm{c}} / \mathrm{AF}_{\mathrm{pd}}\right)$

$\mathrm{m}=1 / \mathrm{b}_{\mathrm{c}}$

em que q é o coeficiente de dano relativo; $m$ é a perda máxima da produtividade relativa, $b_{c p d}$ e $b_{c}$ são coeficientes $b$ obtidos das equações lineares recíprocas do ensaio em série de substituição ( $c=$ cultura e pd = planta daninha) e da cultura, respectivamente; $\mathrm{AF}_{\mathrm{c}}$ e $\mathrm{AF}_{\mathrm{pd}}$ são áreas foliares da cultura e da competidora, respectivamente, ambas provenientes do ensaio conduzido em monoculturas. Os valores obtidos dos parâmetros q e m foram utilizados como inicializadores do procedimento Proc Nlin do programa SAS (Equações 2 e 3).

\section{Resultados e Discussão}

O modelo original de população que estima perdas de produtividade com base em características morfológicas mostrou valores significativos da estatística $\mathrm{F}$ em todas as situações testadas (Tabela 1). De modo geral, os dados ajustaram-se adequadamente ao modelo, com $\mathrm{R}^{2}$ médio de 0,739 . No entanto, os ajustes médios para as cultivares diferiram, demonstrando haver alguma influência relacionada ao desenvolvimento ou ao tipo de planta na grandeza do ajuste. Da mesma forma, considerando-se os valores médios de $\mathrm{R}^{2}$, a área foliar tendeu a ajustar melhor o modelo do que cobertura foliar ou massa seca. Obteve-se melhor ajuste geral no espaçamento de $25 \mathrm{~cm}$, comparativamente ao de $15 \mathrm{~cm}$.

Os valores estimados do parâmetro i aumentaram com o incremento do espaçamento entrelinhas, com exceção de algumas situações relacionadas à área foliar (Tabela 1). O parâmetro i é um índice utilizado na comparação da competitividade relativa entre espécies (Swinton et al., 1994). Assim, constatou-se que a redução do espaçamento entrelinhas $(15 \mathrm{~cm})$ aumentou a competitividade das cultivares e reduziu a da cultivar simuladora de arroz-vermelho. A comparação entre cultivares, quanto ao parâmetro i, demonstra incremento gradativo na diferença entre espaçamentos entrelinhas. À medida que aumentou o ciclo de desenvolvimento das cultivares, alcançou-se maior benefício pela redução no espaçamento na cultivar de ciclo mais longo. Quanto ao parâmetro a, na maioria das situações, o modelo superestimou a assíntota, sendo necessário restringi-la a $100 \%$ de perda. Esses resultados podem decorrer das populações máximas da competidora terem sido insuficientes para alcançar perda real máxima de produtividade.

O modelo sugerido por Kropff \& Spitters (1991) para estimar perdas de produtividade, com base nas variáveis relativas, apresentou valores de $\mathrm{F}$ significativos em todas as situações avaliadas (Tabela 2). Ajustes satisfatórios dos dados ao modelo foram constatados, obtendo-se valor médio de $\mathrm{R}^{2}$ de 0,752 . Como no modelo anterior, constatou-se acréscimo no valor médio de $\mathrm{R}^{2}$ à medida que aumentou o ciclo de desenvolvimento das cultivares. Quanto às três variáveis explicativas, houve poucas diferenças, nos ajustes do modelo; na média geral, a variável área foliar relativa foi a que apresentou maior valor de $\mathrm{R}^{2}$.

Em geral, o aumento do espaçamento entrelinhas incrementou o valor estimado do parâmetro q (Tabela 2). Segundo Kropff \& Spitters (1991), quando a cultura e as plantas daninhas apresentam características fisiológicas e morfológicas semelhantes e a cultura desenvolve-se numa população em que a produtividade do monocultivo alcança seu valor máximo, o valor do coeficiente de dano relativo (q) se aproxima de 1. 
Na condição em que a planta daninha é competidora que 1, mas quando a cultura for mais competitiva que a mais forte que a cultura, o valor do parâmetro será maior planta daninha, o valor de q será menor que 1.

Tabela 1. Ajustes ao modelo de população para perda da produtividade de grãos em cultivares de arroz por interferência da cultivar EEA 406, simuladora de arroz-vermelho, em função do espaçamento entrelinhas e em resposta a variáveis avaliadas na cultivar concorrente. IRGA/Cachoeirinha, RS, 2000/2001.

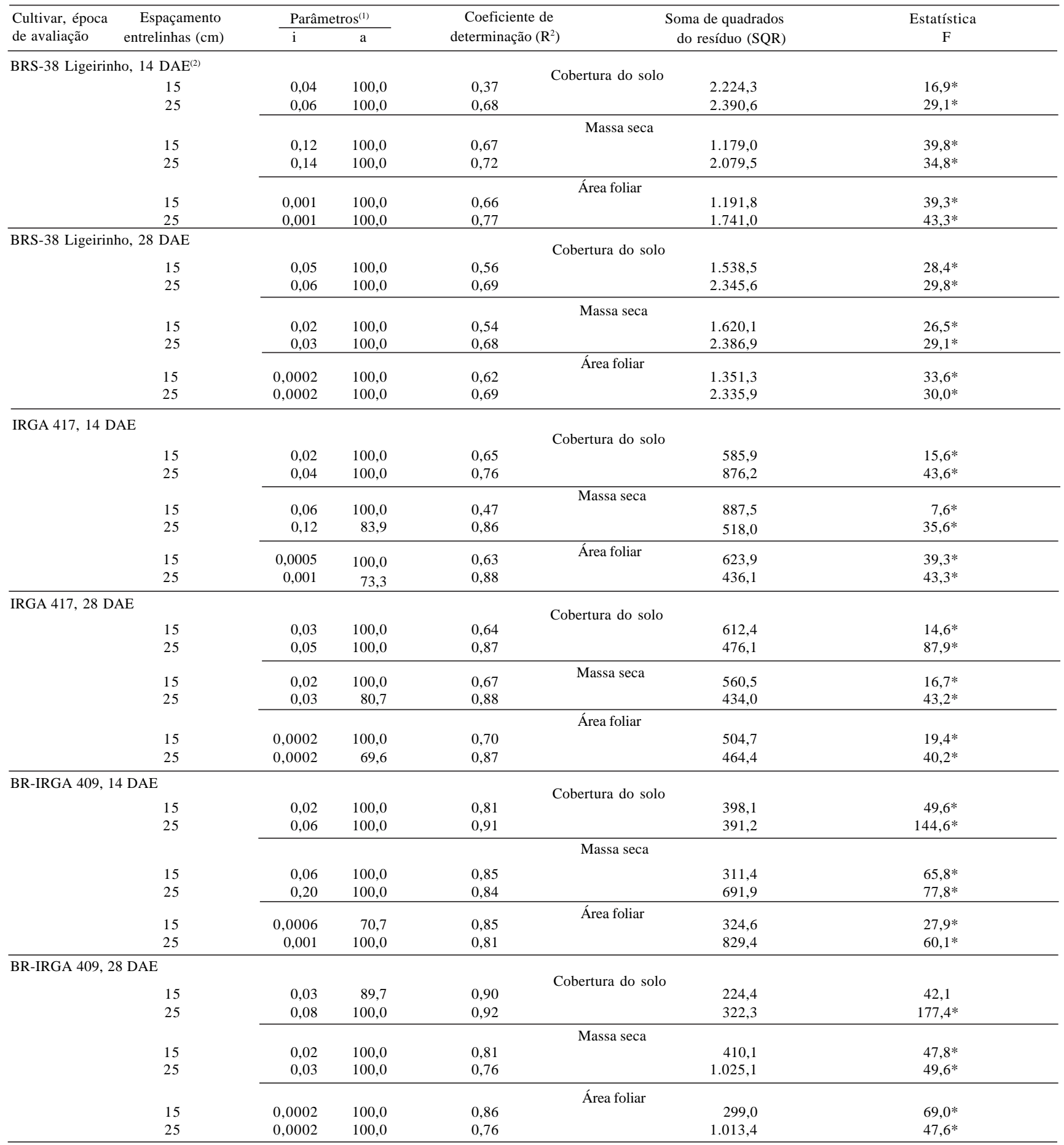

(1)i e a: perdas de produtividade $(\%)$ por unidade da cultivar simuladora quando o valor da variável se aproxima de zero ou tende ao infinito, respectivamente. ${ }^{(2)}$ Dias após a emergência do arroz. *Significativo a $5 \%$ de probabilidade. 
As variáveis relativas cobertura do solo e massa seca geralmente apresentaram valores de q superiores a 1 no maior espaçamento entrelinhas, indicando que a culti- var simuladora foi mais competitiva do que as demais (Tabela 2). Ao contrário, em relação à área foliar relativa, indiferentemente de espaçamento entrelinhas, as

Tabela 2. Ajustes ao modelo de um parâmetro para perda da produtividade de grãos em cultivares de arroz por influência da cultivar EEA 406, simuladora de arroz-vermelho, em função do espaçamento entrelinhas e em resposta a variáveis relativas. IRGA/Cachoeirinha, RS, 2000/2001.

\begin{tabular}{|c|c|c|c|c|c|}
\hline $\begin{array}{c}\text { Cultivar, época } \\
\text { de avaliação }\end{array}$ & $\begin{array}{l}\text { Espaçamento } \\
\text { entrelinhas }(\mathrm{cm})\end{array}$ & $\begin{array}{c}\text { Parâmetros } \\
\mathrm{q}^{(1)} \\
\end{array}$ & $\begin{array}{c}\text { Coeficiente de } \\
\text { determinação }\left(\mathrm{R}^{2}\right)\end{array}$ & $\begin{array}{r}\text { Soma de quadrados } \\
\text { do resíduo (SQR) } \\
\end{array}$ & \multirow[t]{2}{*}{$\begin{array}{c}\text { Estatística } \\
\mathrm{F} \\
\end{array}$} \\
\hline \multicolumn{5}{|c|}{ BRS-38 Ligeirinho, $14 \mathrm{DAE}^{(2)}$} & \\
\hline & 15 & 0,97 & 0,50 & $1.788,1$ & $23,2 *$ \\
\hline & 25 & 1,06 & 0,63 & $2.769,4$ & $23,9 *$ \\
\hline & $\begin{array}{l}15 \\
25\end{array}$ & $\begin{array}{l}0,87 \\
1,01\end{array}$ & $\begin{array}{l}0,61 \\
0,67\end{array}$ & $\begin{array}{l}1.372,7 \\
2.479,6\end{array}$ & $\begin{array}{l}32,9 * \\
27,7 *\end{array}$ \\
\hline & 15 & 0,09 & 0,69 & $1.108,5$ & $42,9 *$ \\
\hline & 25 & 0,10 & 0,73 & $2.028,8$ & $35,9 *$ \\
\hline \multicolumn{6}{|c|}{ BRS-38 Ligeirinho, 28 DAE } \\
\hline & 15 & 1,90 & 0,63 & $1.316,3$ & $34,8^{*}$ \\
\hline & 25 & 1,53 & 0,68 & $2.421,5$ & $28,6^{*}$ \\
\hline & & & & & \\
\hline & 15 & 1,25 & 0,59 & $1.462,0$ & $30,4^{*}$ \\
\hline & 25 & 1,23 & 0,66 & $2.541,1$ & $26,8^{*}$ \\
\hline & & & & & \\
\hline & 15 & 0,10 & 0,61 & $1.377,4$ & $32,8^{*}$ \\
\hline & 25 & 0,12 & 0,67 & $2.493,5$ & $27,5^{*}$ \\
\hline \multirow{2}{*}{\multicolumn{6}{|c|}{ IRGA 417,14 DAE }} \\
\hline & & & & & \\
\hline & 15 & 0,55 & 0,68 & 544,6 & $17,4 *$ \\
\hline & 25 & 0,88 & 0,76 & 872,2 & $43,9 *$ \\
\hline & & & & & \\
\hline & 15 & 0,44 & 0,68 & 539,7 & $17,6^{*}$ \\
\hline & 25 & 1,06 & 0,88 & 414,8 & $102,2 *$ \\
\hline & & & & & \\
\hline & 15 & 0,04 & 0,66 & 574,4 & $16,1^{*}$ \\
\hline & 25 & 0,07 & 0,88 & 446,6 & $94,3^{*}$ \\
\hline \multirow[t]{7}{*}{ IRGA $417,28 \mathrm{D}$} & & & & & \\
\hline & 15 & 1,19 & 0,68 & 541,8 & $17,5^{*}$ \\
\hline & 25 & 1,26 & 0,87 & 455,9 & $92,2 *$ \\
\hline & 15 & 1,00 & 0,68 & 540,5 & $17,6^{*}$ \\
\hline & 25 & 1,13 & 0,87 & 477,0 & $87,7 *$ \\
\hline & 15 & 0,08 & 0,70 & 497,9 & $19,8 *$ \\
\hline & 25 & 0,08 & 0,85 & 538,8 & $76,6^{*}$ \\
\hline \multirow[t]{9}{*}{ BR-IRGA 409,1} & DAE & & & & \\
\hline & 15 & 0,76 & 0,82 & 397,5 & $49,6^{*}$ \\
\hline & 25 & 1,05 & 0,90 & 446,4 & $119,5^{*}$ \\
\hline & & & & & \\
\hline & 15 & 0,57 & 0,85 & 320,9 & $63,6^{*}$ \\
\hline & 25 & 1,20 & 0,90 & 452,4 & $117,8^{*}$ \\
\hline & & & & & \\
\hline & 15 & 0,04 & 0,82 & 389,3 & $50,9^{*}$ \\
\hline & 25 & 0,10 & 0,90 & 415,9 & 128,9 \\
\hline \multicolumn{6}{|c|}{ BR-IRGA 409, 28 DAE } \\
\hline & & & & & \\
\hline & 15 & 1,26 & 0,89 & 226,2 & 94,0 \\
\hline & 25 & 1,86 & 0,89 & 462,7 & 115,0 \\
\hline & & & & & \\
\hline & 15 & 1,06 & 0,84 & 353,4 & $56,9^{*}$ \\
\hline & 25 & 1,20 & 0,77 & 994,8 & 48,7 \\
\hline & & & & & \\
\hline & 15 & 0,10 & 0,86 & 302,7 & $68,0^{*}$ \\
\hline & 25 & 0,13 & 0,77 & 995,4 & 48,6 \\
\hline
\end{tabular}

${ }^{(1)}$ Coeficiente de dano relativo. ${ }^{(2)}$ Dias após a emergência do arroz. *Significativo a 5\% de probabilidade. 
cultivares mostraram ser potencialmente mais competitivas que a cultivar simuladora de arroz-vermelho.

Os valores estimados do parâmetro q na segunda avaliação superaram os da primeira (Tabela 2). Isso parece indicar que a taxa de crescimento da área foliar da cultura foi relativamente menor que a da cultivar concorrente durante o período. Segundo Kropff \& Spitters (1991), ocorrência de mudanças no valor de q associam-se com a taxa de crescimento de folhas durante o período de crescimento exponencial das plantas. Os resultados deste trabalho permitem inferir que alterações nos valores do coeficiente de dano relativo, em função da época de avaliação, variam com as características próprias dos competidores.

O modelo de variáveis relativas (um parâmetro) elevou apenas $1,8 \%$ o valor médio de $\mathrm{R}^{2}$, comparativamente ao modelo de população, demonstrando ser mais adequado para estimar perdas de produtividade por interferência em arroz irrigado. Em relação às três variáveis explicativas, ocorreu pouco ganho com adoção deste modelo, apenas houve algum benefício quanto à variável massa seca. Nos espaçamentos entrelinhas, houve divergência nos resultados, ou seja, o modelo de um parâmetro ajustou melhor os dados do espaçamento de $15 \mathrm{~cm}$, enquanto o modelo de população ajustou melhor os dados do espaçamentos de $25 \mathrm{~cm}$.

A inclusão da perda máxima de produtividade relativa $(\mathrm{m})$ ao modelo de estimativa de perda de produtividade, conforme proposto por Lotz et al. (1996), apresentou ajuste satisfatório dos dados, e a estatística $\mathrm{F}$ foi significativa em todas as situações avaliadas (Tabela 3). $\mathrm{O}$ valor médio de $\mathrm{R}^{2}$ com aplicação deste modelo foi de 0,809 e o aumento do espaçamento entrelinhas elevou os valores de $\mathrm{R}^{2}$. Com poucas exceções, os maiores valores de m estiveram mais associados ao espaçamento entrelinhas de $25 \mathrm{~cm}$ do que ao de $15 \mathrm{~cm}$, especialmente quando se considera a variável área foliar. Igualmente, maiores valores de $\mathrm{m}$ tenderam a ocorrer na cultivar BR-IRGA 409. Em geral, maiores valores de $\mathrm{m}$ também estiveram associados à variável cobertura do solo do que às outras duas.

A inclusão do parâmetro $m$ ao modelo de perda de produtividade que considera variáveis relativas, em geral, reduziu os valores estimados do parâmetro q, comparativamente ao modelo de um único parâmetro. Segundo Kropff \& Spitters (1991), resultados que apresentam esse comportamento indicam situações em que a cultura é forte competidora com a espécie daninha associada. Além disso, os resultados obtidos podem decorrer do fato de o parâmetro $m$ definir claramente uma assíntota, sendo comumente necessário sua inclusão nos conjuntos de dados contendo baixas perdas máximas de produtividade. Porém, mesmo em situação em que as perdas máximas de produtividade, decorrentes da interferência de plantas daninhas, foram elevadas, como é o caso deste trabalho, a utilização do modelo de dois parâmetros melhorou o ajuste.

Em geral, os valores estimados do parâmetro q aumentaram com o incremento do espaçamento entrelinhas (Tabela 3). Esses resultados corroboram os obtidos com o modelo de um parâmetro, em que o aumento do espaçamento entrelinhas diminuiu a habilidade competitiva das cultivares em relação à cultivar competidora. Também os valores de q aumentaram numericamente da primeira para a segunda avaliação efetuada, demonstrando que o atraso na quantificação das variáveis diminui a habilidade competitiva das cultivares. Da mesma forma, observaram-se valores de q consideravelmente maiores das variáveis cobertura do solo e massa seca do que da área foliar, indicando que as estimativas da habilidade competitiva em arroz são modificadas pela variável explicativa considerada.

Os ajustes dos dados ao modelo de dois parâmetros (q e m) foram melhores, comparativamente aos outros dois modelos referidos anteriormente. Na média, aquele modelo aumentou em 9,5 e 7,6\% os valores de $\mathrm{R}^{2}$, em relação aos modelos de população e ao de um parâmetro, respectivamente. Os ganhos alcançados com o modelo de dois parâmetros foram especialmente mais relevantes nos casos em que um dos dois outros modelos mostrou menores ajustes. Do mesmo modo, os ajustes relativos aos espaçamentos entrelinhas foram melhores com o modelo de dois parâmetros, comparativamente aos demais. Houve maior vantagem com o modelo de dois parâmetros em relação à variável explicativa cobertura do solo, quando comparado ao ajuste obtido com o modelo de população. Por sua vez, em relação à variável explicativa área foliar, o modelo de dois parâmetros se mostrou menos vantajoso no ajuste dos dados.

A comparação entre os modelos que descrevem as perdas de produtividade de grãos de arroz, tendo por base variáveis relativas, demonstra que o modelo de dois parâmetros estimou melhor a perda de produtividade, comparativamente ao modelo de um parâmetro (Tabela 4). Em todos os casos estudados, o modelo de dois parâmetros apresentou menores $\mathrm{SQR}$ e, em média, elevou em 7,6\% o valor do $\mathrm{R}^{2}$. No entanto, das 36 comparações realizadas, somente em sete (cerca de $20 \%$ dos casos) o modelo que incluiu a perda máxima de produtividade $(\mathrm{m})$ apresentou aumentos significativos nos ajustes. Dieleman et al. (1995), Lotz et al. (1996) e Lutman et al. (1996) obtiveram resultados semelhantes. 
$\mathrm{O}$ ajuste dos dados experimentais foi melhor com a mente ao modelo de população (Tabela 4). Das 36 comutilização do modelo de dois parâmetros, comparativaparações realizadas com base na SQR, o modelo de

Tabela 3. Ajustes ao modelo de dois parâmetros para perda da produtividade de grãos em cultivares de arroz por interferência de cultivar EEA 406, simuladora de arroz-vermelho, em função do espaçamento entrelinhas e em resposta a variáveis relativas. IRGA/Cachoeirinha, RS, 2000/2001.

\begin{tabular}{|c|c|c|c|c|c|c|}
\hline \multirow{2}{*}{$\begin{array}{l}\text { Cultivar, época } \\
\text { de avaliação }\end{array}$} & \multirow{2}{*}{$\begin{array}{c}\text { Espaçamento } \\
\text { entrelinhas }(\mathrm{cm})\end{array}$} & \multicolumn{2}{|c|}{ Parâmetros ${ }^{(1)}$} & \multirow{2}{*}{$\begin{array}{c}\text { Coeficiente de } \\
\text { determinação }\left(\mathrm{R}^{2}\right)\end{array}$} & \multirow{2}{*}{$\begin{array}{l}\text { Soma de quadrados } \\
\text { do resíduo (SQR) }\end{array}$} & \multirow{2}{*}{$\begin{array}{c}\text { Estatística } \\
\mathrm{F}\end{array}$} \\
\hline & & $\mathrm{q}$ & $\mathrm{m}$ & & & \\
\hline \multicolumn{7}{|c|}{ BRS-38 Ligeirinho, $14 \mathrm{DAE}^{(2)}$} \\
\hline & 15 & 0,77 & 1,66 & 0,83 & 565,7 & $42,5^{*}$ \\
\hline & 25 & 0,91 & 1,27 & 0,64 & $2.745,6$ & $10,7 *$ \\
\hline & $\begin{array}{l}15 \\
25\end{array}$ & $\begin{array}{l}0,58 \\
0,72\end{array}$ & $\begin{array}{l}2,05 \\
2,66\end{array}$ & $\begin{array}{l}0,63 \\
0,85\end{array}$ & $\begin{array}{l}1.286,1 \\
1.136,0\end{array}$ & $\begin{array}{l}15,9^{*} \\
31,6^{*}\end{array}$ \\
\hline & 15 & 0,12 & 1,09 & 0,69 & $1.103,7$ & $19,2^{*}$ \\
\hline & 25 & 0,08 & 1,51 & 0,75 & $1.869,9$ & $17,6^{*}$ \\
\hline \multicolumn{7}{|c|}{ BRS-38 Ligeirinho, 28 DAE } \\
\hline & 15 & 0,99 & 1,73 & 0,91 & 311,9 & $78,0^{*}$ \\
\hline & 25 & 0,83 & 2,85 & 0,83 & $1.204,0$ & $28,9 *$ \\
\hline & 15 & 0,98 & 1,32 & 0,59 & $1.446,1$ & $13,7 *$ \\
\hline & 25 & 0,73 & 2,59 & 0,91 & 649,5 & $48,6^{*}$ \\
\hline & & & & & & \\
\hline & 15 & 0,12 & 0,91 & 0,61 & $1.371,2$ & $14,7^{*}$ \\
\hline & 25 & 0,09 & 1,00 & 0,75 & $1.906,2$ & $17,2 *$ \\
\hline \multicolumn{7}{|c|}{ IRGA 417,14 DAE } \\
\hline & & & & & & \\
\hline & 15 & 0,27 & 1,62 & 0,76 & 403,3 & $11,5^{*}$ \\
\hline & 25 & 0,58 & 2,46 & 0,85 & 546,7 & $33,5^{*}$ \\
\hline & & & & & & \\
\hline & 15 & 0,31 & 1,59 & 0,75 & 377,4 & $12,5^{*}$ \\
\hline & 25 & 0,87 & 1,25 & 0,89 & 380,9 & $49,8^{*}$ \\
\hline & & & & & & \\
\hline & 15 & 0,02 & 0,38 & 0,74 & 434,5 & $10,4^{*}$ \\
\hline & 25 & 0,11 & 0,72 & 0,89 & 390,8 & $48,5^{*}$ \\
\hline \multicolumn{7}{|c|}{ IRGA 417, 28 DAE } \\
\hline & 15 & 0,54 & 2,07 & 0,76 & 397,5 & $11,7 *$ \\
\hline & 25 & 0,97 & 1,81 & 0,88 & 430,9 & $43,6^{*}$ \\
\hline & 15 & 0,53 & 1,09 & 0,80 & 295,9 & $15,4^{*}$ \\
\hline & 25 & 0,88 & 1,16 & 0,88 & 419,9 & $44,8^{*}$ \\
\hline & & & & & & \\
\hline & 15 & 0,04 & 0,54 & 0,72 & 474,7 & $9,3^{*}$ \\
\hline & 25 & 0,12 & 0,69 & 0,86 & 493,9 & $37,5^{*}$ \\
\hline \multicolumn{7}{|c|}{ BR-IRGA 409, 14 DAE } \\
\hline & 15 & 0,65 & 1,42 & 0,85 & 322,9 & $28,1^{*}$ \\
\hline & 25 & 0,55 & 1,00 & 0,85 & 321,2 & $28,3^{*}$ \\
\hline & & & & & & \\
\hline & 15 & 0,70 & 0,70 & 0,85 & 312,9 & $29,1 *$ \\
\hline & 25 & 0,86 & 1,98 & 0,91 & 385,4 & $62,1 *$ \\
\hline & & & & & & \\
\hline & 15 & 0,04 & 1,18 & 0,82 & 388,7 & $22,6^{*}$ \\
\hline & 25 & 0,06 & 4,45 & 0,94 & 275,2 & $88,6^{*}$ \\
\hline \multicolumn{7}{|c|}{ BR-IRGA 409, 28 DAE } \\
\hline & & & & & & \\
\hline & 15 & 1,00 & 1,08 & 0,93 & 157,0 & $62,1^{*}$ \\
\hline & 25 & 1,37 & 1,49 & 0,94 & 248,9 & $98,4 *$ \\
\hline \multicolumn{7}{|c|}{ Massa seca } \\
\hline & 15 & 0,86 & 2,53 & 0,84 & 339,7 & $26,5^{*}$ \\
\hline & 25 & 0,97 & 1,64 & 0,77 & 962,7 & $22,5^{*}$ \\
\hline \multicolumn{7}{|c|}{ Área foliar } \\
\hline & 15 & 0,08 & 0,89 & 0,86 & 300,8 & $30,4^{*}$ \\
\hline & 25 & 0,09 & 2,30 & 0,78 & 930,8 & $23,4^{*}$ \\
\hline
\end{tabular}

${ }^{(1)} \mathrm{q}$ : coeficiente de dano relativo; m: perda máxima de produtividade relativa. ${ }^{(2)}$ Dias após a emergência do arroz. $*$ Significativo a $5 \%$ de probabilidade.

Pesq. agropec. bras., Brasília, v.39, n.9, p.847-856, set. 2004 
dois parâmetros foi superior em 28 situações (78\%). Com em 26 casos (72\%). O modelo com base em variáveis relação ao $\mathrm{R}^{2}$, o modelo de dois parâmetros foi superior foliares relativas apresenta vantagens em relação aos

Tabela 4. Comparação entre modelos para estimar perdas de produtividade de grãos em arroz irrigado por interferência da cultivar EEA 406, simuladora de arroz-vermelho, com base em variáveis explicativas. IRGA/Cachoeirinha, RS, 2000/2001.

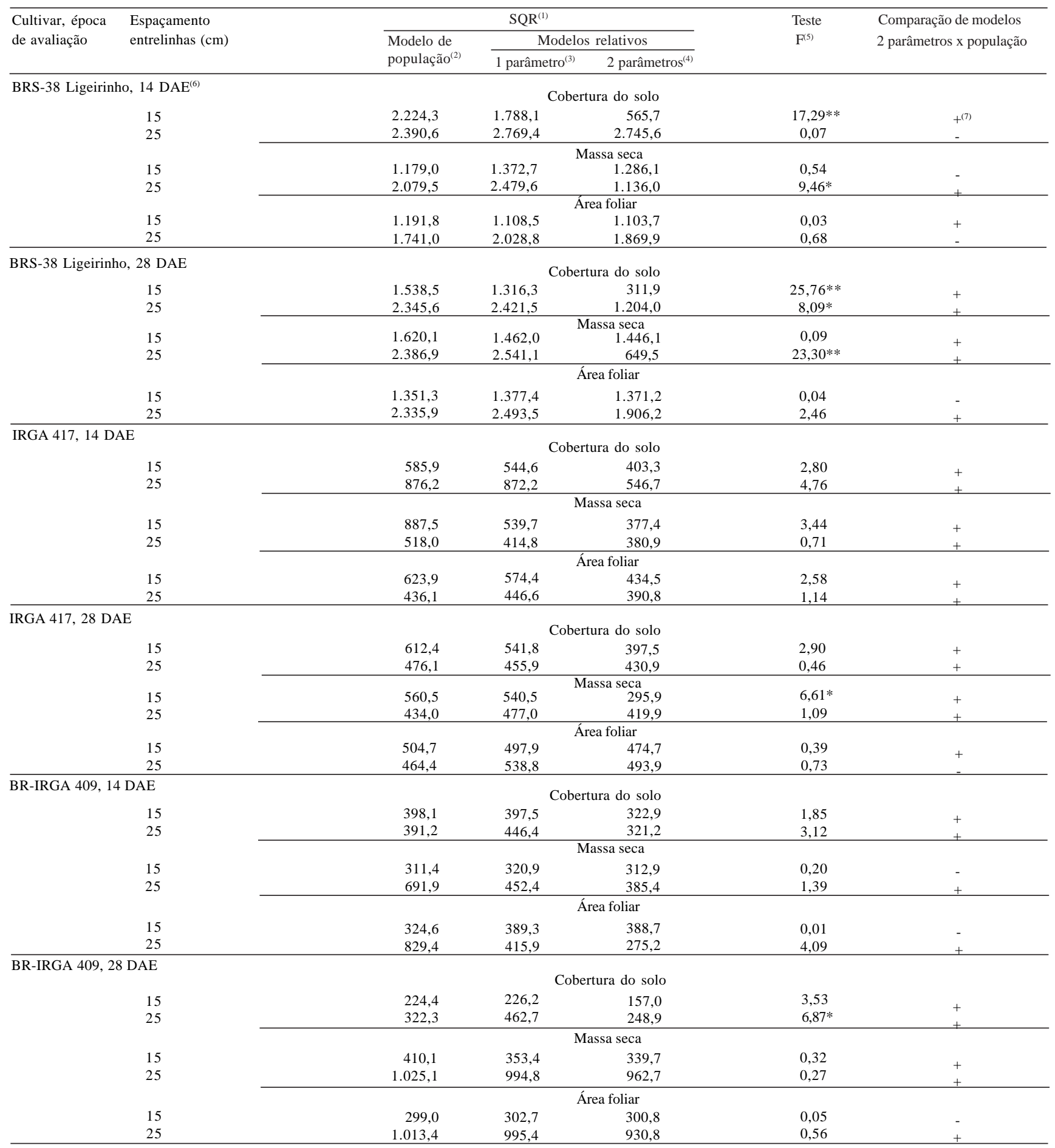

${ }^{(1)}$ Soma de quadrados do resíduo. $\left.{ }^{(2)} \mathrm{Pr}=\left(\mathrm{i}^{*} \mathrm{X}\right) /(1+(\mathrm{i} / \mathrm{a}) \mathrm{X})\right) .{ }^{\left({ }^{3}\right)} \mathrm{Pr}=(100 \mathrm{qX}) /\left(1+\left((\mathrm{q} / \mathrm{m})^{-1}\right) \mathrm{X}\right) .{ }^{(4)} \mathrm{Pr}=(100 \mathrm{qX}) /(1+((\mathrm{q} / \mathrm{m})-1) \mathrm{X}) .{ }^{(5)} \mathrm{Calculado}$ com a Equação 4. ${ }^{(6)}$ Dias após a emergência do arroz. ${ }^{(7)}+$ ou - representam as situações em que o modelo de dois parâmetros foi superior ou inferior, respectivamente, ao modelo de população. * e **Significativo a $5 \%$ e a $1 \%$ de probabilidade. 
modelos que consideram as variáveis população de plantas e épocas relativas de emergência (Kropff \& Spitters, 1991; Chikoye \& Swanton, 1995; Knezevic et al., 1995). Lotz et al. (1996) observaram que, de 23 comparações realizadas, o modelo de dois parâmetros superou o modelo de populações em 21 casos (91\%). Segundo esses autores, o modelo de população mostrou descrição inferior dos dados porque não considerou a época de emergência relativa das plantas daninhas, quando comparado aos modelos de área foliar.

A análise comparativa dos modelos, com base nos graus de associação entre $\mathrm{R}^{2}$ e SQR, apresentou valores de correlação de $-0,59,-0,66$ e $-0,67$, respectivamente, nos modelos de população (Cousens, 1985), de um (Kropff \& Spitters, 1991) e de dois (Lotz et al., 1996) parâmetros. Quanto maior o valor negativo da correlação, melhor é o ajuste dos dados ao modelo. Assim, esses resultados revelam que os modelos com base em variáveis relativas foram superiores ao modelo de população, corroborando os resultados descritos anteriormente.

\section{Conclusões}

1. O modelo de dois parâmetros ajusta melhor os dados do que o modelo de um parâmetro e ambos são superiores ao modelo de população.

2. As variáveis área foliar e cobertura do solo estimam com precisão similar as perdas de produtividade de grãos em arroz irrigado por efeito de interferência da cultivar simuladora.

3. Os modelos testados indicam que a redução do espaçamento entrelinhas aumenta a habilidade competitiva das cultivares de arroz em relação a competidora.

\section{Agradecimento}

Ao CNPq, pela concessão de bolsa aos autores Nilson Gilberto Fleck e Paulo Régis Ferreira da Silva.

\section{Referências}

ASKEW, S.D.; WILCUT, J.W. Tropic croton interference in cotton. Weed Science, v.49, p.184-189, 2001.
CHIKOYE, D.; SWANTON, C.J. Evaluation of three empirical models depicting Ambrosia artemisiifolia competition in white bean. Weed Research, v.35, p.421-428, 1995.

COUSENS, R. An empirical model relating crop yield to weed and crop density and a statistical comparison with other models. Journal of Agricultural Science, v.105, p.513-521, 1985.

DIELEMAN, A.; HAMILL, A.S.; WEISE, S.F.; SWANTON, C.J. Empirical models of pigweed (Amaranthus spp.) interference in soybean (Glycine max). Weed Science, v.43, p.612-618, 1995.

EMBRAPA CLIMA TEMPERADO. Arroz irrigado: recomendações técnicas da pesquisa para o Sul do Brasil. Pelotas: Embrapa Clima Temperado; Irga; Epagri, 1999. 124p. (Embrapa Clima Temperado. Documentos, 57).

KNEZEVIC, S.Z.; WEISE, S.F.; SWANTON, C.J. Comparison of empirical models depicting density of Amaranthus retroflexus L. and relative leaf area as predictors of yield loss in maize (Zea mays L.). Weed Research, v.35, p.207-214, 1995.

KROPFF, M.J.; SPITTERS, C.J.T. A simple model of crop loss by weed competition from early observations on relative leaf area of weeds. Weed Research, v.31, p.97-105, 1991.

LOTZ, L.A.P.; CHRISTENSEN, S.; CLOUTIER, D.; FERNANDEZ QUINTANILLA, C.; LÉGÈRE, A.; LEMIEUX, C.; LUTMAN, P.J.; PARDO IGLESIAS, A.; SALONEN, J.; SATTIN, M.; STIGLIANI, L.; TEI, F. Prediction of the competitive effects of weeds on crop yields based on the relative leaf area of weeds. Weed Research, v.36, p.93-101, 1996.

LUTMAN, P.J.W.; BOWERMAN, P.; PALMER, G.M.; WHYTOCK, G.P. Prediction of competition between oilseed rape and Stellaria media. Weed Research, v.40, p.255-269, 2000.

LUTMAN, P.J.W.; RISIOTT, R.; OSTERMANN, H.P. Investigations into alternative methods to predict the competitive effects of weeds on crop yields. Weed Science, v.44, p.290-297, 1996.

NGOUAJIO, M.; LEMIEUX, C.; FORTIER, J.J.; CAREAU, D.; LEROUX, G.D. Validation of an operator-assisted module to measure weed and crop leaf cover by digital image analysis. Weed Technology, v.12, p.446-453, 1998

RADOSEVICH, S.R.; HOLT, J.S.; GHERSA, C. Weed ecology: implications for management. 2.ed. New York: Wiley, 1997. 588p.

RATKOWSKY, D.A. Nonlinear regression modeling: a unified practical approach. New York: M. Dekker, 1983. p.135-154

SAS INSTITUTE (Cary, Estados Unidos). Statistical analysis system user's guide: version 6. 4th ed. Cary, 1989. 846p.

SWINTON, S.M.; BUHLER, D.D.; FORCELLA, F.; GUNSOLUS, J.L.; KING, R.P. Estimation of crop yield loss due to interference by multiple weed species. Weed Science, v.42, p.103-109, 1994.

YENISH, J.P.; DURGAN, B.R.; MILLER, D.W.; WYSE, D.L. Wheat (Triticum aestivum) yield reduction from common milkweed (Asclepias syriaca) competition. Weed Science, v.45, p.127-131, 1997.

Recebido em 5 de novembro de 2003 e aprovado em 6 de maio de 2004 